\title{
Clinical consequences of chest tube malposition in trauma resuscitation: single-center experience
}

\author{
Manuel F. Struck ${ }^{1}$ (D) Sebastian Ewens ${ }^{2}$. Johannes K. M. Fakler ${ }^{3} \cdot$ Gunther Hempel $^{1}$ - André Beilicke ${ }^{4}$. \\ Michael Bernhard ${ }^{4} \cdot$ Patrick Stumpp $^{2} \cdot$ Christoph Josten $^{3} \cdot$ Sebastian N. Stehr ${ }^{1} \cdot$ Hermann Wrigge $^{1}$. \\ Sebastian Krämer ${ }^{5}$
}

Received: 12 January 2018 / Accepted: 28 May 2018 / Published online: 31 May 2018

(c) Springer-Verlag GmbH Germany, part of Springer Nature 2018

\begin{abstract}
Purpose Evaluation of trauma patients with chest tube malposition using initial emergency computed tomography $(\mathrm{CT})$ and assessment of outcomes and the need for chest tube replacement.

Methods Patients with an injury severity score $>15$, admitted directly from the scene, and requiring chest tube insertion prior to initial emergency CT were retrospectively reviewed. Injury severity, outcomes, and the positions of chest tubes were analyzed with respect to the need for replacement after CT.

Results One hundred seven chest tubes of 78 patients met the inclusion criteria. Chest tubes were in the pleural space in $58 \%$ of cases. Malposition included intrafissural positions (27\%), intraparenchymal positions (11\%) and extrapleural positions (4\%). Injury severity and outcomes were comparable in patients with and without malposition. Replacement due to malfunction was required at similar rates when comparing intrapleural positions with both intrafissural or intraparenchymal positions ( 11 vs. $23 \%, p=0.072$ ). Chest tubes not reaching the target position (e.g., pneumothorax) required replacement more often than targeted tubes ( 75 vs. $45 \%, p=0.027$ ). Out-of-hospital insertions required higher replacement rates than resuscitation room insertions ( 29 vs. $10 \%, p=0.016$ ). Body mass index, chest wall thickness, injury severity, insertion side and intercostal space did not predict the need for replacement.

Conclusions Patients with malposition of emergency chest tubes according to CT were not associated with worse outcomes compared to patients with correctly positioned tubes. Early emergency chest CT in the initial evaluation of severely injured patients allows precise detection of possible malposition of chest tubes that may require immediate intervention.
\end{abstract}

Keywords Chest tube $\cdot$ Multiple trauma $\cdot$ Computed tomography $\cdot$ Tracheal intubation

Manuel F. Struck and Sebastian Ewens are equal contributors.

Manuel F. Struck

manuelstruck@web.de

1 Department of Anesthesiology and Intensive Care Medicine, University Hospital Leipzig, Liebigstr. 20, 04103 Leipzig, Germany

2 Department of Diagnostic and Interventional Radiology, University Hospital Leipzig, Liebigstr. 20, 04103 Leipzig, Germany

3 Department of Orthopedics, Traumatology and Plastic Surgery, University Hospital Leipzig, Liebigstr. 20, 04103 Leipzig, Germany

4 Emergency Department, University Hospital Leipzig, Liebigstr. 20, 04103 Leipzig, Germany

5 Department of Visceral, Transplant, Thoracic and Vascular Surgery, University Hospital Leipzig, Liebigstr. 20, 04103 Leipzig, Germany

\section{Introduction}

Chest tube insertion for the emergency management of severe chest trauma is a key procedure in the Advanced Trauma Life Support ${ }^{\circledR}\left(\right.$ ATLS $\left.^{\circledR}\right)$ algorithm and is included in current guideline recommendations [1,2]. Recently, data from a large national trauma database revealed chest tube placement rates of $8 \%$ in the prehospital setting and $27 \%$ in the early hospital resuscitation phase for patients with an injury severity score $>15$ [3]. Chest tube placement under emergency conditions leads to a high risk for procedural and positional complications, which may occur in up to $38 \%$ of cases [4-8]. Malposition may include intrafissural, intraparenchymal, extrapleural and extrathoracic positions, whereas malfunction may occur in intrapleural positions due to kinking. The lateral approach within the "triangle of 
safety" using blunt dissection rather than a trocar is known to be associated with reduced insertion-related complications $[1,4,5,9]$. In contrast to the traditional notion that chest tubes should ideally be placed close to target structures, current data suggest that sufficient drainage of larger collections of air and/or fluid may be possible from any position within the pleural space [6]. Although the potential for chest tube malposition and the need for early tube position control are known issues in trauma care, studies using computed tomography (CT) for precise analysis of chest tube position are scarce. The aim of this study was to analyze chest tube positions in severely injured patients on initial chest CT. We hypothesized that chest tubes (1) not reaching target structures and (2) considered malpositioned would be associated with higher rates of more severe injuries, worse outcomes and chest tube replacement compared to chest tubes close to target structures and/or with a correct pleural position.

\section{Methods}

After approval from the ethics committee of the Medical Faculty of Leipzig, Germany (No. 137-15-20042015), the local trauma registry of the University Hospital Leipzig was reviewed for patients admitted between 01/2010 and $12 / 2015$. The inclusion criteria were age $>14$ years, admission directly from the scene of the accident, injury severity score (ISS) $>15$, chest tube placement in the out-of-hospital emergency setting or during management in the resuscitation room prior to emergency CT, and available CT data. Data were obtained from medical records, the radiological information system, and the picture archiving and communication system (MEDOS RIS version 9.3.3008, Nexus MagicWeb Version VA60C_0115, Visage Imaging, PACS: syngo.plaza, Siemens Healthcare).

\section{General management}

Out-of-hospital emergency treatment of trauma patients is performed by physician-staffed emergency medical services (EMS) until hospital admission. In our center, resuscitation room management for severely injured patients is organized according to the recommendations of the German Society of Trauma Surgery (DGU) with an interdisciplinary trauma team and the standardized $A T L S^{\circledR}$ approach. Airway management and chest tube placement after admission to the resuscitation room are performed by anesthetists and trauma surgeons, respectively. All procedures are performed by consultants or by residents and specialists under direct supervision of consultants. In prehospital EMS and the resuscitation room, chest tube sizes of 24 and $28 \mathrm{~F}$ are available. Early emergency CT (within the first hour of admission) is routinely performed, whereas patients receiving cardiopulmonary resuscitation or with obvious life-threatening injuries may be transferred directly to the operating room for immediate surgery. In this study, we analyzed emergency CT data for chest tube positions with regard to chest tube replacement immediately after $\mathrm{CT}$ and within the first $24 \mathrm{~h}$ after admission (Fig. 1). Immediate chest tube replacement was performed in cases of persistent pneumothorax and hemothorax or in cases of severe tube malfunction in a patient in critical condition. Delayed replacement within $24 \mathrm{~h}$ after admission was performed due to tube dislocation, to improve the target position, and for additional tube placement for large collections and/or during emergency thoracotomy. Chest tube positions were classified according to a systematic classification using anatomical structures. Achievement of target structure position was defined as direct contact between the chest tube and the pneumothorax and/or collection of blood/fluid as confirmed by CT. If no target could be identified on CT, then the chest tube was classified as reaching the target. Analysis and measurements were performed by a radiologist, an anesthetist and a thoracic surgeon.

\section{Statistical analysis}

The data are reported as the mean \pm standard deviation and as numbers (percentages). Statistical comparisons were performed between chest tubes in the target position vs. not in the target position, pleural position vs. non-pleural position, and the need for replacement immediately after CT and within $<24 \mathrm{~h}$ after admission using the $\chi^{2}$ test for qualitative data, and Student's $t$ test or the Mann-Whitney $U$ test for quantitative data. Patient-centric analysis was performed in overall patients and in a subgroup of patients who underwent only one chest tube placement. Therefore, investigated parameters included chest abbreviated injury severity (AIS) score, ISS, ventilator days, length of stay in the intensive care unit (LOS ICU), 24-h mortality and 30-day mortality. The investigated risk factors of chest tube position-centric analysis were body mass index (BMI), chest wall thickness, chest AIS score, ISS, out-of-hospital or resuscitation room placement, insertion side and intercostal space. The alpha level of significance was set at 0.05 . All tests were two tailed. Multivariate analysis was not performed due to low sample sizes. The analysis was performed using SPSS 20.0, IBM Corp. Armonk, NY, USA.

\section{Results}

During the study period, 2159 trauma team activations occurred, including activations for 526 patients who presented with an ISS $>15$ and direct admission from the scene. 
Fig. 1 Study flow chart

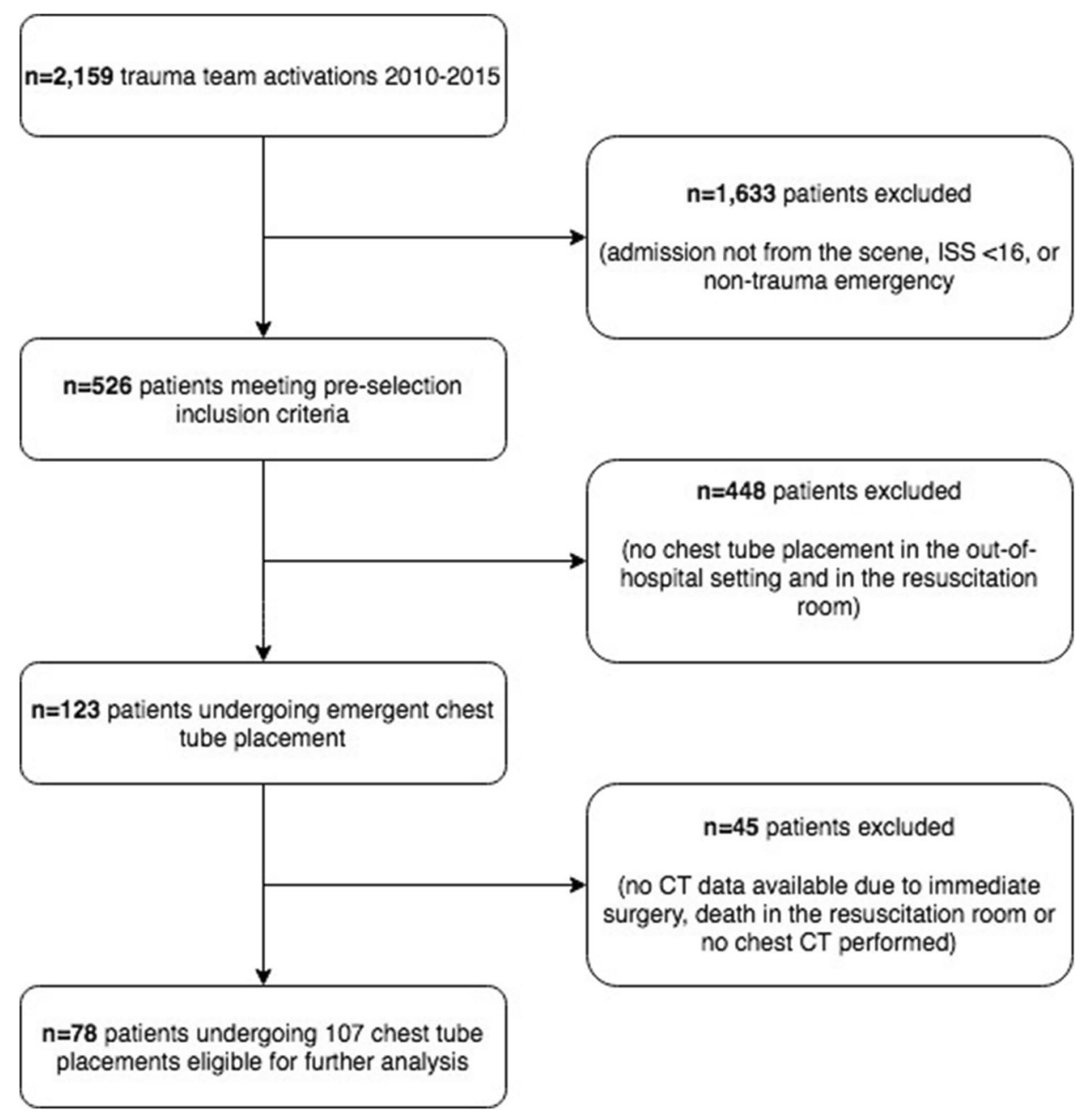

Of these, 123 patients underwent chest tube placement, and 45 patients without chest CT data were excluded (14 deceased in the resuscitation room, 18 underwent immediate surgery, and 13 underwent cranial CT only). 78 patients with 107 chest tube placements had available chest CT data and were further analyzed (Fig. 1). Most patients were male ( $n=58,74 \%$ ), with a mean age of $47 \pm 18$ years, a mean ISS of $36 \pm 15$ and mean AIS chest of $3.9 \pm 0.8$. Additional patient characteristics are displayed in Tables 1, 2, 3, 4 and 5. Blunt trauma mechanisms accounted for $95 \%$ of the injuries (50\% road traffic accidents, $37 \%$ falls, and $8 \%$ other blunt trauma mechanisms) and $5 \%$ of the injuries were caused by penetrating trauma mechanisms. Chest tube placement was performed in the out-of-hospital setting in 30 patients (39\%) and in the resuscitation room in 59 patients $(76 \%)$ prior to CT diagnostic evaluation, whereas 11 patients (14\%) underwent chest tube placement in both the out-of-hospital and the in-hospital settings. Single chest tube placement was performed in 55 patients $(71 \%)$, bilateral tube placement was performed in 19 patients (24\%), and multiple (three and four) chest tubes were placed in four patients $(5 \%)$. Tracheal
Table 1 Demographic data

\begin{tabular}{ll}
\hline Parameter & \\
\hline Age, years, mean \pm SD & $47 \pm 18$ \\
Male, $n(\%)$ & $58(74)$ \\
ISS, mean \pm SD & $36 \pm 15$ \\
AIS chest, mean \pm SD & $3.9 \pm 0.8$ \\
ICU days, mean \pm SD & $13.5 \pm 18.3$ \\
Ventilator days, mean \pm SD & $6.5 \pm 10.7$ \\
24-h mortality, $n(\%)$ & $12(15)$ \\
30-day mortality, $n(\%)$ & $21(27)$ \\
\hline
\end{tabular}

$I S S$ injury severity score, $A I S$ abbreviated injury severity, ICU intensive care unit

intubation prior to chest tube placement was performed in 64 patients (82\%), including $52(67 \%)$ who were intubated in the out-of-hospital setting and $12(15 \%)$ who were intubated in the resuscitation room. Fourteen patients (18\%) underwent chest tube placement awake under local anesthesia. Most chest tubes $(81 \%)$ were placed between the 4 th and 
Table 2 Injury severity and outcomes in overall patients related to chest tube position
Table 3 Injury severity and outcomes of $n=55$ patients with single chest tube insertion related to chest tube position

\begin{tabular}{lllllll}
\hline Parameter & Intrapleural & Not intrapleural & $p$ & In target & Not in target & $p$ \\
\hline ISS, mean \pm SD & $37 \pm 14$ & $36 \pm 14$ & 0.739 & $36 \pm 13$ & $38 \pm 15$ & 0.652 \\
AIS chest, mean \pm SD & $3.9 \pm 0.7$ & $3.8 \pm 0.8$ & 0.455 & $3.9 \pm 0.8$ & $3.9 \pm 0.7$ & 0.627 \\
ICU days, mean \pm SD & $12.2 \pm 18.3$ & $14.3 \pm 17.2$ & 0.544 & $10.1 \pm 12.6$ & $16.1 \pm 21.5$ & 0.080 \\
Ventilator days, mean \pm SD & $5.4 \pm 9.3$ & $8.2 \pm 12.1$ & 0.212 & $5.2 \pm 8.4$ & $7.9 \pm 12.3$ & 0.193 \\
24-h mortality, $n(\%)$ & $13(12.1)$ & $8(7.5)$ & 0.682 & $12(15.7)$ & $9(11.8)$ & 0.495 \\
30-day mortality, $\%$ & $18(16.8)$ & $13(12.1)$ & 0.987 & $17(15.9)$ & $14(13.1)$ & 0.564 \\
\hline
\end{tabular}

$I S S$ injury severity score, $A I S$ abbreviated injury severity, ICU intensive care unit

\begin{tabular}{lllllll}
\hline Parameter & Intrapleural & Not intrapleural & $p$ & In target & Not in target & $p$ \\
\hline ISS, mean \pm SD & $35 \pm 17$ & $36 \pm 17$ & 0.710 & $32 \pm 13$ & $36 \pm 17$ & 0.422 \\
AIS chest, mean \pm SD & $3.6 \pm 0.7$ & $3.5 \pm 0.9$ & 0.749 & $3.4 \pm 0.8$ & $3.7 \pm 0.8$ & 0.239 \\
ICU days, mean \pm SD & $13.2 \pm 22.1$ & $16.4 \pm 18.5$ & 0.564 & $11.4 \pm 0.3$ & $17.2 \pm 25.4$ & 0.304 \\
Ventilator days, mean \pm SD & $4.7 \pm 9.0$ & $8.8 \pm 12.7$ & 0.166 & $5.0 \pm 6.7$ & $7.7 \pm 13.4$ & 0.377 \\
24-h mortality, $n(\%)$ & $5(9.1)$ & $3(5.4)$ & 0.863 & $1(1.8)$ & $4(7.3)$ & 0.231 \\
30-day mortality, $n(\%)$ & $7(12.7)$ & $4(7.3)$ & 0.587 & $4(7.3)$ & $7(12.7)$ & 0.498 \\
\hline
\end{tabular}

$I S S$ injury severity score, $A I S$ abbreviated injury severity, $I C U$ intensive care unit 6th intercostal space using the lateral approach (Table 5). The most commonly used tube size was $28 \mathrm{~F}$, which was used in 83 cases $(78 \%)$. In 17 cases $(16 \%), 24-\mathrm{F}$ chest tubes were used and in 6 cases $(5 \%)$, the chest tube size could not be reliably assessed.

We found comparable injury severity and outcomes in patients with and without intrapleural chest tube position and with and without target position, respectively (Tables 2 , $3)$. In the overall analysis, we found a non-significant trend ( $p=0.080)$ for longer ICU stay in patients without targeted chest tubes (Table 2). This trend could not be confirmed in a subset analysis of patients with single-chest tube insertion $(p=0.304)$ (Table 3).

Chest tube replacement was required prior to $\mathrm{CT}$ diagnostic evaluation in three cases and in 30 cases after CT diagnostic evaluation (16 cases immediately after CT and in another 14 cases within $24 \mathrm{~h}$ after admission). Two patients underwent both immediate and delayed correction of chest tube position. Kinking was observed in 10 cases (9\%). Seven patients underwent additional contralateral chest tube placement after CT within the first $24 \mathrm{~h}$ after admission. According to $\mathrm{CT}$ analysis, chest tubes were in the pleural space in $58 \%$ of cases, whereas malposition included intrafissural positions $(27 \%)$, intraparenchymal positions (11\%) and extrapleural positions (4\%) (Fig. 2; Table 4). Target structures were directly reached in 54 cases (50\%). Chest tubes in target positions were significantly more frequently positioned in the pleural space ( 76 vs. $40 \%, p<0.001$ ). Furthermore, targeted chest tubes required fewer immediate replacements after CT diagnostic evaluation compared to chest tubes not reaching a target ( 7 vs. $23 \%, p=0.027$ ), although no statistical significance was observed in the overall replacement rate (19 vs. $33 \%, p=0.069)$ (Table 5). Chest tubes in intrapleural positions had similar immediate and overall replacement rates compared to those of chest tubes in intrafissural and intraparenchymal positions (11 vs. $23 \%, p=0.072$ and 23 vs. $31 \%, p=0.322$, respectively). Chest tubes requiring replacement immediately after CT ( $n=16 ; 15 \%)$ or within $24 \mathrm{~h}$ after admission $(n=28 ; 26 \%$, including patients with replacements immediately after CT) were located in intrapleural $(n=6,38 \%$ and $n=15$, $56 \%$, respectively), intrafissural $(n=2,13 \%$ and $n=5,19 \%$, respectively), intraparenchymal $(n=4,25 \%$ and $n=4,14 \%$, respectively), and extrapleural $(n=4,25 \%$ and $n=4,14 \%$, respectively) positions (Fig. 2).

Out-of-hospital chest tube placement was significantly associated with immediate and overall replacement (29 vs. $10 \%, p=0.016$ and 50 vs. $18 \%, p<0.001$, respectively) compared to resuscitation room placement. Insertion side, intercostal space, ISS, chest AIS, BMI, and chest wall thickness were not associated with target position, pleural position, or any replacement (Table 5).

\section{Discussion}

We found that less than two-thirds of chest tubes were located in the pleural space and only every second tube had direct contact with target structures according to CT analysis. However, regarding injury severity and outcomes, patients were comparable with and without chest tube malposition. Chest tubes requiring immediate replacement 
Table 4 Classification of chest tube position distribution related to anatomical structures

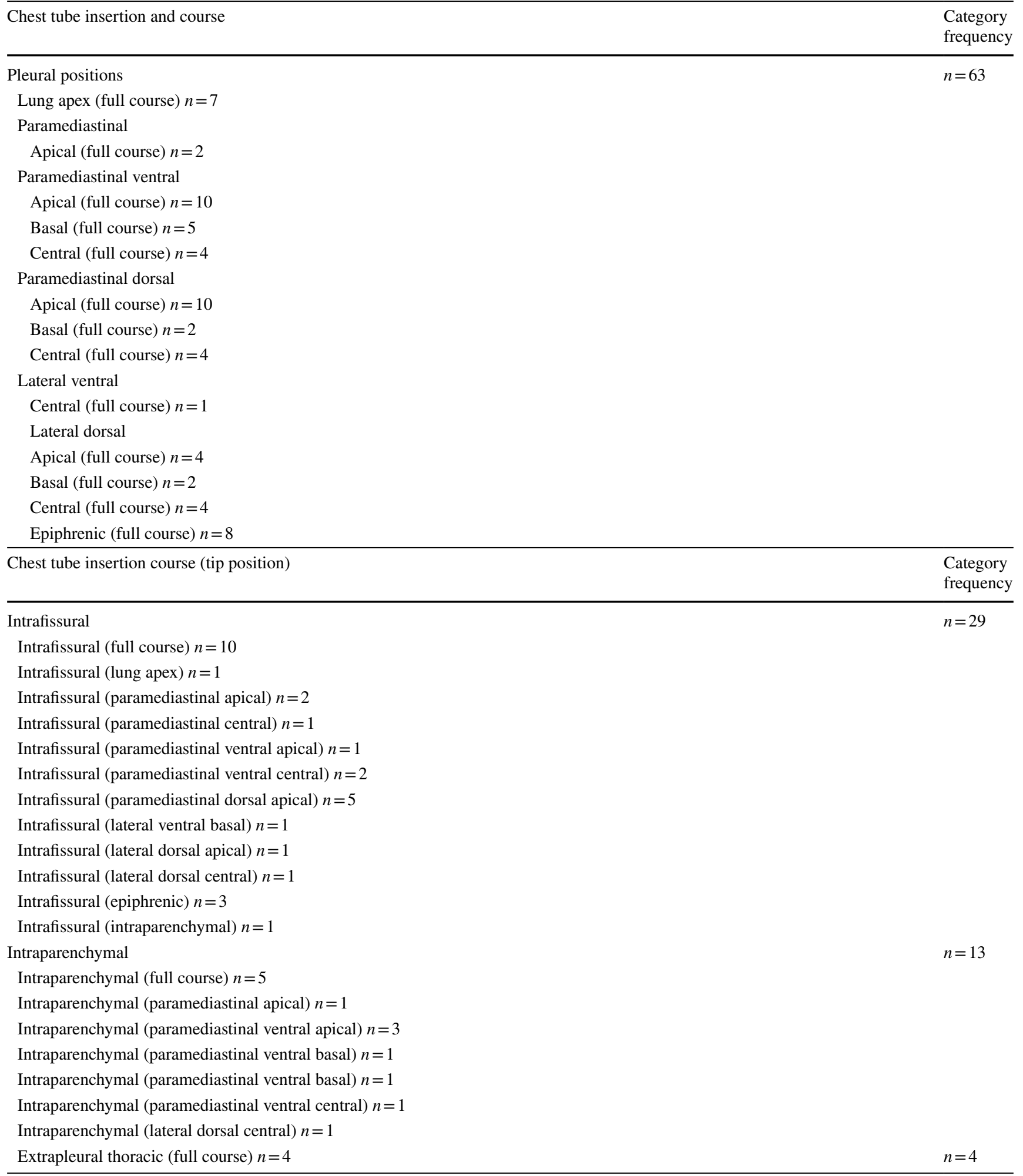

after CT were significantly associated with a failed target position (e.g., persistent pneumothorax), whereas overall tube replacement was required regardless of tube position. Previous studies have suggested that a targeted tube location was not mandatory for functionality as long as the tube was positioned appropriately in the pleural space $[6,10,11]$. Interestingly, intrafissural positions and intraparenchymal positions were not associated with a higher 
Table 5 Need for chest tube replacement and associated risk factors

\begin{tabular}{|c|c|c|c|c|c|c|c|}
\hline Parameter & All & No replacement & $\begin{array}{l}\text { Replacement } \\
\text { immediately after } \\
\text { CT }\end{array}$ & $p$ value & No replacement & $\begin{array}{l}\text { Any replace- } \\
\text { ment within } \\
24 \mathrm{~h}\end{array}$ & $p$ value \\
\hline Insertion side & & & & 0.377 & & & 0.773 \\
\hline Right side, $n(\%)$ & $56(52.3)$ & $46(50.5)$ & $10(62.5)$ & & $42(53.2)$ & $14(50.0)$ & \\
\hline Left side, $n(\%)$ & $51(47.7)$ & $45(49.5)$ & $6(37.5)$ & & $37(46.8)$ & $14(50.0)$ & \\
\hline Intercostal space & & & & 0.750 & & & 0.730 \\
\hline $1, n(\%)$ & $2(1.9)$ & $2(2.2)$ & 0 & & $2(2.5)$ & 0 & \\
\hline $2, n(\%)$ & $3(2.8)$ & $2(2.2)$ & $1(7.1)$ & & $2(2.5)$ & $1(3.8)$ & \\
\hline $3, n(\%)$ & $8(7.6)$ & $8(8.8)$ & 0 & & $8(10.1)$ & 0 & \\
\hline $4, n(\%)$ & 27 (25.7) & $24(26.4)$ & $3(21.4)$ & & $18(22.8)$ & $9(34.6)$ & \\
\hline $5, n(\%)$ & $32(36.2)$ & $28(30.8)$ & $4(28.6)$ & & $24(30.4)$ & $8(30.8)$ & \\
\hline $6, n(\%)$ & $28(26.7)$ & $22(24.2)$ & $6(42.9)$ & & $21(26.6)$ & 7 (27.0) & \\
\hline $7, n(\%)$ & $3(2.8)$ & $3(3.3)$ & 0 & & $2(2.5)$ & $1(3.8)$ & \\
\hline $8, n(\%)$ & $1(0.9)$ & $1(1.1)$ & 0 & & $1(1.3)$ & 0 & \\
\hline $9, n(\%)$ & $1(0.9)$ & $1(1.1)$ & 0 & & $1(1.3)$ & 0 & \\
\hline $\mathrm{ISS}$, mean $\pm \mathrm{SD}$ & $36 \pm 15$ & $34 \pm 13$ & $37 \pm 15$ & 0.239 & $34 \pm 13$ & $36 \pm 14$ & 0.412 \\
\hline Chest AIS score, mean \pm SD & $3.9 \pm 0.8$ & $3.9 \pm 0.7$ & $4.2 \pm 0.8$ & 0.197 & $3.9 \pm 0.8$ & $4.1 \pm 0.7$ & 0.316 \\
\hline Body mass index, mean \pm SD & $27.4 \pm 5.1$ & $27.1 \pm 4.9$ & $26.8 \pm 2.7$ & 0.523 & $27.4 \pm 5.1$ & $26.1 \pm 3.2$ & 0.065 \\
\hline Chest wall thickness, $\mathrm{cm}$, mean $\pm \mathrm{SD}$ & $4.9 \pm 2.3$ & $4.8 \pm 2.3$ & $4.6 \pm 1.6$ & 0.663 & $4.9 \pm 2.3$ & $4.4 \pm 1.9$ & 0.310 \\
\hline Target position & & & & 0.027 & & & 0.069 \\
\hline Yes, $n(\%)$ & $54(50.5)$ & $50(54.9)$ & $4(25.0)$ & & $44(55.7)$ & $10(35.7)$ & \\
\hline No, $n(\%)$ & $53(49.5)$ & $41(45.1)$ & $12(75.0)$ & & $35(44.3)$ & $18(64.3)$ & \\
\hline Position intrapleural & & & & 0.072 & & & 0.322 \\
\hline Yes, $n(\%)$ & $62(58.0)$ & $56(61.5)$ & $6(37.5)$ & & $48(60.8)$ & $14(50.0)$ & \\
\hline No, $n(\%)$ & $45(42.0)$ & $35(38.5)$ & $10(62.5)$ & & $31(39.2)$ & $14(50.0)$ & \\
\hline Insertion environment & & & & 0.019 & & & $<0.001$ \\
\hline Out-of-hospital & $28(26.2)$ & $20(22.0)$ & $8(50.0)$ & & $14(17.8)$ & $14(50.0)$ & \\
\hline Resuscitation room & $79(73.8)$ & $71(78.0)$ & $8(50.0)$ & & $65(82.2)$ & $14(50.0)$ & \\
\hline
\end{tabular}

ISS injury severity score, $A I S$ abbreviated injury severity

rate of position correction compared with pleural positions, possibly because only 11 of 29 intrafissural tubes (33\%) and 4 of 11 intraparenchymal tubes $(36 \%)$ were entirely located in these positions, whereas most of the tubes' terminal ends were in pleural positions. Conversely, tube drainage may be sufficient when only the terminal end is in the intrafissural or intraparenchymal position and proximal holes provide access to the pleural space. One study with comparable sample sizes concluded that the most frequent malposition (17 of 22 cases) was intrafissural, whereas functional malposition, which was defined as radiological malposition clinically requiring repositioning, occurred in only six cases (6\%) [5]. Although our data are consistent with the literature that intrafissural and intraparenchymal positions are not necessarily associated with the need for replacement, [6, 9, 10], any undetected malfunction may delay intended pleural decompression or hematoma drainage and may, therefore, be potentially life threatening and increase risks of secondary interventions and costs [12].

Emergency circumstances of trauma resuscitation always elicit uncertainty regarding correct indications and the ability of the operator to place a targeted tube without proper visual guidance. The decision for chest tube placement must be determined within a small time window based on the clinical condition of a patient and according to unspecific surrogate parameters (e.g., attenuated breath sounds and subcutaneous emphysema). Out-of-hospital performance of emergency procedures is often negatively influenced by a hostile environment (e.g., noise, improper light, confusing situations, and various simultaneous measures). In our study, out-of-hospital insertion was significantly associated with immediate and later replacement compared to resuscitation room placement. This finding probably reflects the complexity of the emergency setting and/or the higher risk for possible tube displacement due to transport. However, 
Fig. 2 Chest tube position and need for replacement

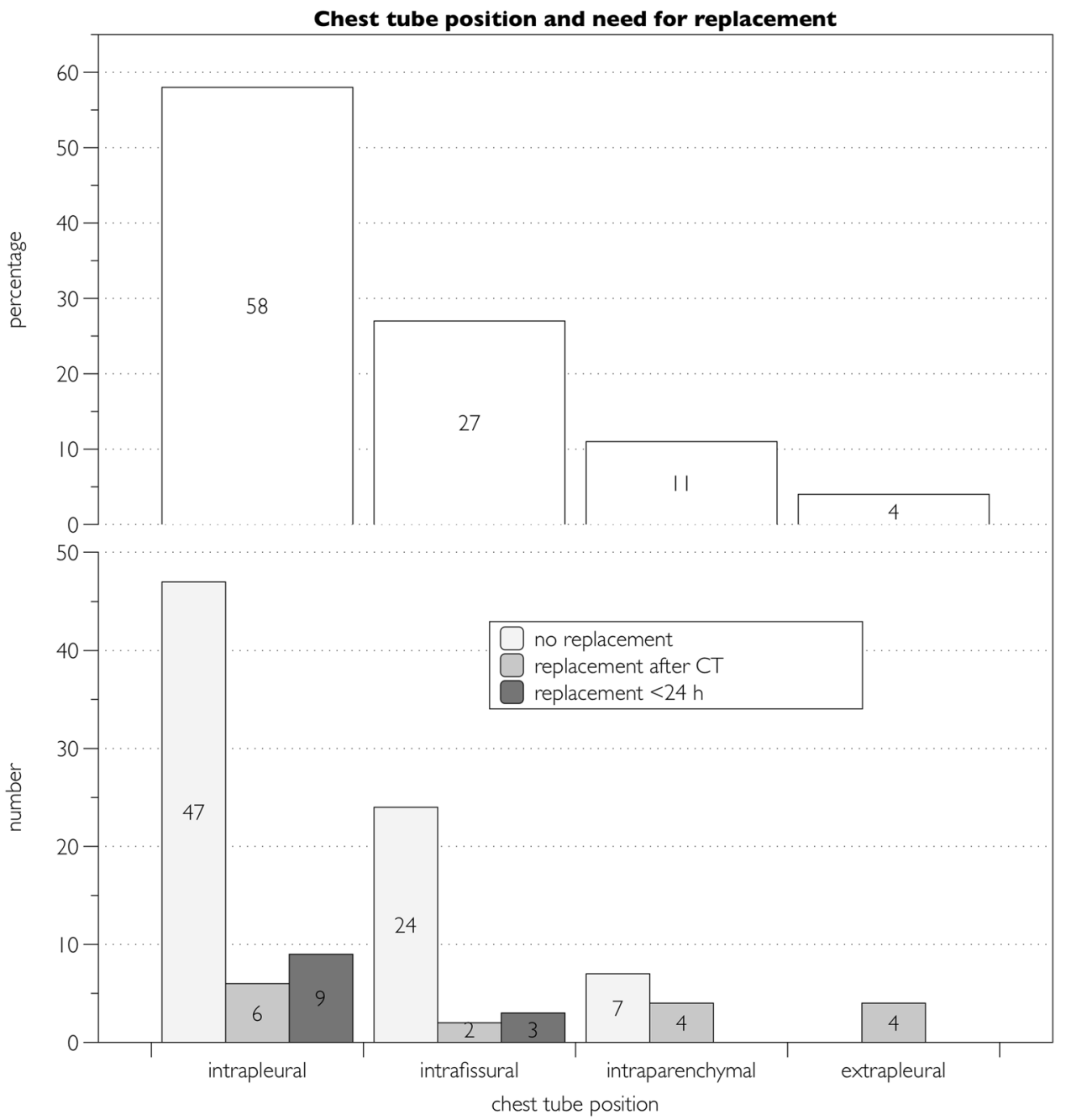

other studies did not find relevant differences in complication rates for out-of-hospital chest tube placement compared to resuscitation room placement [5, 7]. Furthermore, overall resuscitation times (the time from the accident until the end of resuscitation room treatment) were comparable between out-of-hospital vs. resuscitation room chest tube placement, although out-of-hospital resuscitation times were longer in patients who underwent out-of-hospital chest tube placement [13]. Published risk factors for chest tube complications (e.g., right side insertions, intercostal space level, chest AIS score, body mass index, and chest wall thickness) were not associated with either immediate or overall chest tube replacement in our study. In the literature, high-quality prospective, randomized studies with large sample sizes of chest tube placements are not available in the setting of trauma resuscitation. Furthermore, studies are often not comparable due to different definitions and heterogeneity among study populations. Many studies include a considerable proportion of penetrating injury patients in contrast to only $5 \%$ in our study $[6,8]$. A recent study investigated the need for secondary intervention after chest tube placement and identified higher chest AIS scores, penetrating injury mechanism, and initial drainage volume of hemothorax as significant risk factors [6]. Chest AIS score had previously been confirmed in other studies as a prognostic parameter for the development of chest tube complications [14]. In a study with a high proportion of trauma patients, only use of a trocar (a risk factor that we could not analyze in the current study) was identified as a robust risk factor for tube malposition, which occurred in $30 \%$ of patients. The authors highlighted that CT diagnostic evaluation of tube position should consider radiologist consultation because neither clinical nor radiologic signs are sensitive enough to appropriately detect chest tube malposition [4]. Our data confirm that the actual position of a chest tube measured using CT cannot reliably predict its clinical functionality. The dichotomy among the published literature regarding the need for a targeted tube position for appropriate functionality should be explored in further studies.

We acknowledge the general limitations of retrospective studies. Findings can only be interpreted as associations rather than causative relations. We only included patients 
who underwent $\mathrm{CT}$ following chest tube placement. Patients who died before CT evaluation may have suffered severe chest tube-related complications that could potentially contribute to fatality, as shown in autopsy or postmortem CT studies $[15,16]$. The indication for chest tube replacement may be subjective and may not be always based on functionality and/or radiologic position. This makes it difficult to classify the incidence of reposition as really necessary or not. However, immediate repositioning after CT may be associated with higher probability of real necessity for repositioning (e.g., due to persistent tension pneumothorax or large fluid collections). We could not assess the size of the chest tubes for all patients due to the retrospective nature of the study. Although $28-\mathrm{F}$ chest tubes were used in most cases, a reliable correlation between tube size and incidence of malposition was not possible in this study. However, one study confirmed that chest tube size did not impact the clinically relevant outcomes tested [17]. Furthermore, we were not able to obtain outcome data regarding pneumonia, wound infection, and empyema in this study, which may have influenced outcome. In addition, the level of training of emergency physicians could not be assessed and may have varied considerably compared to that of physicians working in resuscitation rooms.

\section{Conclusions}

Patients with malposition of emergency chest tubes according to CT were not associated with worse outcomes compared to patients with correctly positioned tubes. Intrafissural and intraparenchymal positions required similar replacement rates due to malfunction compared with intrapleural positions. Non-targeted chest tubes and out-of-hospital insertions were associated with higher replacement rates. Early emergency chest CT in the initial evaluation of severely injured patients allows precise detection of possible malposition of chest tubes that may require immediate intervention.

Author contributions MFS, SE, and SK contributed to conception and design; MFS, SE, JKMF, GH, AB, MB, SNS and HW analyzed and interpreted the data; MFS, SE, GH and AB collected data; MFS drafted the article; MFS, SE, JKMF, GH, AB, MB, PS, CJ, SNS, HW, and SK critically revised the article for important intellectual content.

Funding There was no funding for this study.

\section{Compliance with ethical standards}

Ethical approval All procedures performed in studies involving human participants were in accordance with the ethical standards of the institutional and/or national research committee and with the 1964 Helsinki declaration and its later amendments or comparable ethical standards.
Informed consent This was a retrospective study and did not require informed consent. Therefore, the Local Ethics Committee of the Medical Faculty of the University of Leipzig approved the study and waived informed consent.

Conflict of interest Manuel F. Struck, Sebastian Ewens, Johannes K. M. Fakler, Gunther Hempel, André Beilicke, Michael Bernhard, Patrick Stumpp, Christoph Josten, Sebastian N. Stehr, Hermann Wrigge, and Sebastian Krämer declare that they have no conflict of interest.

\section{References}

1. American College of Surgeons. Advanced trauma life support, ATLS $^{\circledR}$ student course manual. 9th ed. Chicago:ACS;2012.

2. AWMF S3-Leitlinie Polytrauma/Schwerverletzten-Behandlung, Registrierungsnummer 012-019, AWMF, Düsseldorf, http://www. awmf.org/leitlinien. Accessed 12 Jan 2018.

3. Bayer J, Lefering R, Reinhardt S, Kühle J, Südkamp NP, Hammer T, TraumaRegister DGU. Severity-dependent differences in early management of thoracic trauma in severely injured patientsanalysis based on the TraumaRegister DGU ${ }^{\circledR}$. Scand J Trauma Resusc Emerg Med. 2017;25:10.

4. Remérand F, Luce V, Badachi Y, Lu Q, Bouhemad B, Rouby JJ. Incidence of chest tube malposition in the critically ill: a prospective computed tomography study. Anesthesiology. 2007;106:1112-9.

5. Huber-Wagner S, Körner M, Ehrt A, Kay MV, Pfeifer KJ, Mutschler W, Kanz KG. Emergency chest tube placement in trauma care-which approach is preferable? Resuscitation. 2007;72:226-33.

6. Benns MV, Egger ME, Harbrecht BG, Franklin GA, Smith JW, Miller KR, Nash NA, Richardson JD. Does chest tube location matter? An analysis of chest tube position and the need for secondary interventions. J Trauma Acute Care Surg. 2015;78:386-90.

7. Maybauer MO, Geisser W, Wolff H, Maybauer DM. Incidence and outcome of tube thoracostomy positioning in trauma patients. Prehosp Emerg Care. 2012;16:237-41.

8. Kong VY, Oosthuizen GV, Sartorius B, Keene C, Clarke DL. An audit of the complications of intercostal chest drain insertion in a high volume trauma service in South Africa. Ann R Coll Surg Engl. 2014;96:609-13.

9. Laws D, Neville E, Duffy J; Pleural Diseases Group. Standards of Care Committee, British Thoracic Society. BTS guidelines for the insertion of a chest drain. Thorax. 2003;58(Suppl 2):ii53-9.

10. Curtin JJ, Goodman LR, Quebbeman EJ, Haasler GB. Thoracostomy tubes after acute chest injury: relationship between location in a pleural fissure and function. AJR Am J Roentgenol. 1994;163:1339-42.

11. Landay M, Oliver Q, Estrera A, Friese R, Boonswang N, DiMaio JM. Lung penetration by thoracostomy tubes: imaging findings on CT. J Thorac Imaging. 2006;21:197-204.

12. Hernandez MC, Zeb MH, Heller SF, Zielinski MD, Aho JM. Tube thoracostomy complications increase Cost. World J Surg. 2017:41:1482-7.

13. Kulla M, Helm M, Lefering R, Walcher F. Prehospital endotracheal intubation and chest tubing does not prolong the overall resuscitation time of severely injured patients: a retrospective, multicentre study of the Trauma Registry of the German Society of Trauma Surgery. Emerg Med J. 2012;29:497-501.

14. Menger R, Telford G, Kim P, Bergey MR, Foreman J, Sarani B, Pascual J, Reilly P, Schwab CW, Sims CA. Complications following thoracic trauma managed with tube thoracostomy. Injury. 2012;43:46-50. 
15. Lotan E, Portnoy O, Konen E, Simon D, Guranda L. The role of early postmortem CT in the evaluation of support-line misplacement in patients with severe trauma. AJR Am J Roentgenol. 2015;204:3-7.

16. Ondruschka B, Baier C, Dreßler J, Höch A, Bernhard M, Kleber C, Buschmann C. Additional emergency medical measures in trauma-associated cardiac arrest. Anaesthesist. 2017;66:924-35.
17. Inaba K, Lustenberger $\mathrm{T}$, Recinos $\mathrm{G}$, Georgiou $\mathrm{C}$, Velmahos GC, Brown C, Salim A, Demetriades D, Rhee P. Does size matter? A prospective analysis of 28-32 versus 36-40 French chest tube size in trauma. J Trauma Acute Care Surg. 2012;72:422-7. 